\title{
A new purely growing instability in a strongly magnetized nonuniform pair plasma
}

\author{
Nitin Shukla ${ }^{a}$ and P. K. Shukla ${ }^{b)}$ \\ Institut für Theoretische Physik IV, \\ Fakultät für Physik und Astronomie, \\ Ruhr-Universität Bochum, D-44780 Bochum, Germany
}

(Dated: Received 9 February 2007)

\begin{abstract}
It is shown that a strongly magnetized nonuniform electron-positron (hereafter referred to as e-p or pair) plasma is unstable against low-frequency (in comparison with the electron gyrofrequency) electrostatic oscillations. For this purpose, a dispersion relation is derived by using the Poisson equation as well as the electron and positron continuity equations with the guiding center drifts for the electron and positron fluids. The dispersion relation admits a purely growing instability in the presence of the equilibrium density and magnetic field inhomogeneities. Physically, instability arises because of the inhomogeneous magnetic field induced differential electron and positron density fluctuations, which do not keep in phase with the electrostatic potential arising from the charge separation in our nonuniform pair plasmas.
\end{abstract}

PACS numbers: PACS number (s): 52.27.Ep, 52.35.Fp, 52.35.Qz 
Pair plasmas are composed of charged particles with same mass and opposite charges. They are ubiquteous in the early universe [1], in pulsar magnetospheres [2-7], in bipolar outflows (jets), in active galactic nuclei [8, 9], in fireballs producing gamma-ray bursts [10], at the center of our own galaxy [11], in solar flares [12], and in inertial confinement fusion scheme using ultraintense lasers [13-16]. Nonrelativistic pair plasmas have also been created in laboratory experiments [17]. Pair production also occurs in tokamaks [18] due to collisions between multi-MeV runaway electrons and thermal particles.

It is well known that pair plasmas admit the time and space scales that significantly differ from those of an electron-ion plasma due to equal masses of the pairs. There appear a great variety of new linear $[19,20]$ and nonlinear $[19,21-26]$ waves in a uniform pair plasma. The understanding of mechanisms for exciting different wave modes in pair plasmas is of fundamental importance, since nonthermal fluctuations can control transport and acceleration of the plasma particles.

In this Brief Report, we present a new purely growing instability for generating electrostatic fluctuations in a strongly magnetized pair plasma containing the equilibrium density and magnetic field gradients. At the equilibrium, we have

$$
\frac{d}{d x}\left[\frac{B_{0}^{2}(x)}{8 \pi}+n_{0}(x) T\right]=0,
$$

where $B_{0}(x)$ is the strength of the external magnetic field, $n_{0}(x)$ is the equilibrium electron number density, and $T=T_{e}+T_{p}$ is the sum of the electron and positron temperatures. The external magnetic field is $\mathbf{B}_{0}=B_{0}(x) \hat{\mathbf{z}}$, where $\hat{\mathbf{z}}$ is the unit vector along the z-direction in a Cartesian coordinate system. The charge-neutrality condition at the equilibrium is $n_{e 0}(x)=n_{p 0}(x)=n_{0}(x)$.

We now study instability of our equilibrium against low-frequency (in comparison with the electron gyrofrequency $\omega_{c}=e B_{0} / m c$, where $e$ is the magnitude of the electron charge, $m$ is the electron/positron mass, and $c$ is the speed of light in vacuum), two-dimensional electrostatic perturbations. The perpendicular (to $\hat{\mathbf{z}}$ ) component of the electron and positron fluid velocities in the electrostatic field $\mathbf{E}_{\perp}=-\nabla_{\perp} \phi$, where $\phi$ is the electrostatic potential, are

$$
\mathbf{u}_{e \perp} \approx \frac{c}{B_{0}} \hat{\mathbf{z}} \times \nabla \phi-\frac{c T_{e}}{e B_{0} n_{e 0}} \hat{\mathbf{z}} \times \nabla n_{e 1}
$$


and

$$
\mathbf{u}_{p \perp} \approx \frac{c}{B_{0}} \hat{\mathbf{z}} \times \nabla \phi+\frac{c T_{p}}{e B_{0} n_{p 0}} \hat{\mathbf{z}} \times \nabla n_{p 1},
$$

where $n_{e 1}\left(\ll n_{e 0}\right)$ and $n_{p 1}\left(\ll n_{p 0}\right)$ are small electron and positron number density perturbations around their equilibrium values. We have neglected the electron and positron polarization drifts in our tenuous strongly magnetized plasma.

The electron and positron number density perturbations are determined from

$$
\frac{\partial n_{e 1}}{\partial t}+\nabla_{\perp} \cdot\left(n_{e 0} \mathbf{u}_{e \perp}\right)=0
$$

and

$$
\frac{\partial n_{p 1}}{\partial t}+\nabla_{\perp} \cdot\left(n_{p 0} \mathbf{u}_{p \perp}\right)=0
$$

The electron and positron density perturbations are coupled with $\phi$ through the Poisson equation

$$
\nabla_{\perp}^{2} \phi=4 \pi e\left(n_{e 1}-n_{p 1}\right)
$$

Substituting (2) and (3) into (4) and (5), we obtain, respectively,

$$
\left(\frac{\partial}{\partial t}-V_{B e} \frac{\partial}{\partial y}\right) n_{e 1}=c \frac{d}{d x}\left(\frac{n_{e 0}}{B_{0}}\right) \frac{\partial \phi}{\partial y}
$$

and

$$
\left(\frac{\partial}{\partial t}+V_{B p} \frac{\partial}{\partial y}\right) n_{p 1}=c \frac{d}{d x}\left(\frac{n_{p 0}}{B_{0}}\right) \frac{\partial \phi}{\partial y}
$$

where $V_{B e}=\left(c T_{e} / e B_{0}\right) d \ln B_{0} / d x$ and $V_{B p}=\left(T_{p} / T_{e}\right) V_{B e}$.

Supposing that $n_{e 1}, n_{p 1}$ and $\phi$ are proportional to $\exp (i k y-i \omega t)$, where $k_{y}$ and $\omega$ are the $y$ component of the wavevector $\mathbf{k}$ and the frequency, respectively, we Fourier transform (6), (7) and (8) to obtain

$$
\begin{aligned}
& \left(\omega+k_{y} V_{B e}\right) n_{e 1}=-k_{y} c \frac{d}{d x}\left(\frac{n_{e 0}}{B_{0}}\right) \phi \\
& \left(\omega-k_{y} V_{B p}\right) n_{p 1}=-k_{y} c \frac{d}{d x}\left(\frac{n_{p 0}}{B_{0}}\right) \phi
\end{aligned}
$$


and

$$
k_{y}^{2} \phi=4 \pi e\left(n_{p 1}-n_{e 1}\right)
$$

where we have assumed that the wavelength $\left(2 \pi / k_{y}\right)$ is much smaller than the scalesizes of the density and magnetic field gradients.

Eliminating from (11) $n_{e 1}$ and $n_{p 1}$ by using (9) and (10), we obtain the dispersion relation for $T_{e}=T_{p}=T_{0}$ and $n_{e 0}=n_{p 0}=n_{0}$

$$
\omega^{2}-k_{y}^{2} V_{B}^{2}+\frac{\omega_{p}^{2}}{\omega_{c}} K_{n b} V_{B}=0,
$$

where $V_{B}=\left(c T_{0} / e B_{0} L_{B}\right), L_{B}=\left(d \ln B_{0} / d x\right)^{-1}$ is the scalelength of the magnetic field gradient, $\omega_{p}=\left(4 \pi n_{0} e^{2} / m\right)^{1 / 2}$ is the electron plasma frequency, and $K_{n b}=d \ln \left(n_{0} / B_{0}\right)$.

Letting $\omega=i \gamma$ in (12), we obtain for the growth rate

$$
\gamma=\left(\frac{\omega_{p}^{2}}{\omega_{c}} K_{n b} V_{B}-k_{y}^{2} V_{B}^{2}\right)^{1 / 2},
$$

which shows that a purely growing instability occurs if

$$
K_{n b}>k_{y}^{2} V_{B} \omega_{c} / \omega_{p}^{2}
$$

The growth rate above threshold is

$$
\gamma=\frac{\rho_{e} \omega_{p}}{\left|L_{n} L_{B}\right|^{1 / 2}}
$$

where $\rho_{e}=V_{T} / \omega_{c}$ is the electron thermal gyroradius, $V_{T}=\left(T_{0} / m\right)^{1 / 2}$ is the electron thermal speed, and $L_{n}=\left(d \ln n_{0} / d x\right)^{-1}$ is the scalelength of the density gradient. Equation (15) has been obtained from (13) by using (1) and (14). Equation (15) reveals an e-folding time of one second for $\rho_{e} /\left|\sqrt{L_{n} L_{B}}\right| \sim 10^{-6}$ and $n_{0} \sim 10^{5} \mathrm{~cm}^{-3}$.

To summarize, we have derived a new dispersion relation for the low-frequency (in comparison with the electron gyrofrequency) electrostatic waves in a nonuniform magnetoplasma containing the equilibrium density and magnetic field inhomogeneities. The dispersion relation admits a purely growing instability. The latter arises due to the magnetic field gradient, which produces differential electron and positron density fluctuations that are out of phase with the electrostatic potential. The present result should be helpful for understanding the 
origin of nonthermal purely growing electrostatic waves and the glitches in strongly magnetized nonuniform pair plasmas, such as those in dense neutron stars, in the Crab Nebula, and in some laboratory experiments.

\section{Acknowledgments}

Nitin Shukla thanks Profs. Luis Silva and Gianfranco Sorasio for their kind support at the Instituto Superior Técnico, Lisboa, Portugal. 
[a)] Also at the Nonlinear Physics Centre \& Centre for Plasma Science and Astrophysics, RuhrUniversität Bochum, D-44780 Bochum, Germany; Grupo de Lasers e Plasmas, Departamento de Física do Instituto Superior Técnico, Universidade Técnica de Lisboa, 1049-001 Lisboa, Portugal; and Department of Physics, Umeå University, SE-90187 Umeå, Sweden. E-mail: ns@tp4.rub.de

[b)] Also at the Nonlinear Physics Centre \& Centre for Plasma Science and Astrophysics, RuhrUniversität Bochum, D-44780 Bochum, Germany; Department of Physics, Umeå University, SE-90187 Umeå, Sweden; Max-Planck-Institut für extraterrestrische Physik, D-85741 Garching, Germany; Grupo de Lasers e Plasmas, Departamento de Física do Instituto Superior Técnico, Universidade Técnica de Lisboa, 1049-001 Lisboa, Portugal; CCLRC Centre for Fundamental Physics, Rutherford Appleton Laboratory, Chilton, Didcot, Oxon 0X11 0QX, UK; SUPA Department of Physics, University of Strathclyde, Glasgow G 40NG, UK; School of Physics, Faculty of Science \& Agriculture, University of KwaZulu-Natal, Durban 4000, South Africa. Email: ps@tp4.rub.de

[1] G. W. Gibbons, S. C. Wilks, and S. Siklos, The Very Early Universe (Cambridge University Press, Cambridge, 1983).

[2] V. L. Ginzburg, Sov. Phys. Usp. 14, 83 (1971).

[3] P. A. Sturrock, Astrophys. J. 164, 529 (1971).

[4] M. A. Ruderman and P. G. Sutherland, Astrophys. J. 196, 51 (1975).

[5] R. N. Manchester and J. H. Taylor, Pulsars (Freeman, San Francisco, 1977).

[6] F. C. Michel, Rev. Mod. Phys. 54, 1 (1982).

[7] F. C. Michel, Theory of Neutron Star Magnetospheres (University of Chicago, Chicago, 1991).

[8] M. C. Begelman, R. D. Blandford, and M. D. Rees, Rev. Mod. Phys. 56, 255 (1984).

[9] H. R. Miller and P. J. Witta, Active Galactic Nuclei (Springer, Berlin, 1987), p. 202.

[10] T. Piran, Phys. Rep. 314, 575 (1999).

[11] M. L. Burns, in Positron-Electron Pairs in Astrophysics, edited by M. L. Burns, A. K. Harding, and R. Ramaty (AIP, New York, 1983).

[12] E. Tandberg-Hansen and A. G. Emshie, The Physics of Solar Flares (Cambridge University Press, Cambridge, 1988), p. 124. 
[13] V. I. Berezhiani, D. D. Tskhakaya and P. K. Shukla, Phys. Rev. A 46, 6608 (1992).

[14] E. P. Liang, S. C. Wilks, and M. Tabak, Phys. Rev. Lett. 81, 4887 (1998); S. C. Wilks, H. Chen, E. Liang et al., Astrophys. Space Sci. 298, 347 (2005).

[15] C. Gahn, G. D. Tsakiris, G. Pretzler et al., Appl. Phys. Lett. 77, 2662 (2000).

[16] M. Marklund and P. K. Shukla, Rev. Mod. Phys. 78, 591 (2006).

[17] R. G. Greaves, M. D. Tinkel, and C. M. Surko, Phys. Plasmas 1, 1439 (1994); C. M. Surko, M. Leventhal, and A. Passner, Phys. Rev. Lett. 62, 901 (1989); R. G. Greaves and C. M. Surko, Phys. Rev. Lett. 75, 3846 (1995); R. G. Greaves and C. M. Surko, Phys. Rev. Lett. 85, $1883(2000)$.

[18] P. Helander and D. J. ward, Phys. Rev. Lett. 75, 3846 (2003).

[19] P. K. Shukla, N. N. Rao, M. Y. Yu, and N. L. Tsintsadze, Phys. Rep. 138, 1 (1986).

[20] N. Iwamoto, Phys. Rev. E 47, 604 (1993).

[21] M. E. Gedalin, J. G. Lominadze, L. Stenflo, and V. N. Tsytovich, Astrophys. Space Sci. 108, 393 (1985).

[22] L. Stenflo, P. K. Shukla and M. Y. Yu, Astrophys. Space Sci. 117, 303 (1985); M. Y. Yu, P. K. Shukla, and L. Stenflo, Astrophys. J. 309, L63 (1986)

[23] R. T. Gangadhara, V. Krishan, and P. K. Shukla, Month. Not. R. Astron. Soc. 262, 151 (1993).

[24] S. I. Popel, S. V. Vladimirov, and P. K. Shukla, Phys. Plasmas 2, 716 (1995).

[25] F. Verheest, M. A. Hellberg, G. J. Gray, and R. L. Mace, Astrophys. Space Sci.239, 125 (1996); F. Verheest, Phys. Lett. A 213, 177 (1996); F. Verheest and T. Cattaert, Phys. Plasmas 11, 3078 (2004); F. Verheest ibid. 13, 082301 (2006).

[26] E. Asseo, Plasma Phys. Controlled Fusion 45, 853 (2003). 